\title{
Dichloroacetate is Promising for Treating Hematological Malignancy through Inhibiting Ketone Bodies Oxidation: towards Better Understanding of Its Anticancer Mechanisms
}

\author{
Mongi Ayat ${ }^{*}$ \\ Division of Hematology, Department of Clinical Biochemistry and Molecular Medicine, Taibah College of Medicine, \\ Taibah University, Al-Madinah Al-Munawwarah, Saudi Arabia \\ *Corresponding author: mongi_64@yahoo.com
}

\begin{abstract}
Dichloroacetate (DCA) is a promising anticancer drug that exerts potent anticancer effects in many clinical oncology studies. On biochemical and pharmacological bases, this article aims at gaining a better understanding of DCA anticancer effects. Ketone bodies oxidation (ketolysis) is an important source of energy to many cancer cells. Here, it is proved that DCA antagonizes acetoacetate and targets cancer cells' energetics through inhibiting ketolysis as novel evidence-based anticancer mechanisms. DCA was reported to inhibit oxidation of both ketone bodies (acetoacetate and $\beta$-hydroxybutyrate) in addition to palmitate. Acetoacetate diverted pyruvate metabolism from pyruvate dehydrogenase (PDH) to pyruvate carboxylation while DCA increased the oxidation of glucose through PDH. This suggests an antagonism between DCA and ketone bodies. Moreover, DCA was reported to inhibit $\beta$-hydroxybutyrate uptake by the extra-splanchnic tissues and decrease the clearance of ketone bodies. That may be explained by structural antagonism between DCA and ketone bodies leading to a competitive uptake at target tissues i.e. DCA may competitively antagonize ketone bodies. In a previous study, DCA infusion in starved rats caused a significant decrease in blood glucose, plasma insulin, blood lactate and pyruvate concentrations but significantly increased concentrations of ketone bodies ( $\beta$-hydroxybutyrate and acetoacetate) (Blackshear et al., 1974). Based on that, DCA inhibits ketone bodies utilization for energy production. In conclusion, DCA enhances anticancer immunity, targets anaerobic cancer cell populations (via targeting Warburg effect) and targets aerobic cancer cell populations through targeting mitochondrial energy generating pathways e.g. ketolysis.
\end{abstract}

Keywords: dichloroacetate, cancer, ketolysis, Warburg effect

Cite This Article: Mongi Ayat, "Dichloroacetate is Promising for Treating Hematological Malignancy through Inhibiting Ketone Bodies Oxidation: towards Better Understanding of Its Anticancer Mechanisms." American Journal of Cancer Prevention, vol. 6, no. 1 (2018): 5-8. doi: 10.12691/ajcp-6-1-2.

\section{Introduction}

DCA is a promising anticancer drug that antagonizes Warburg effect and exerts potent anticancer effects with promising results in clinical oncology. [1] DCA effectively cured a chemoresistant non-Hodgkin's lymphoma patient after disease progression with rituximab-CHOP chemotherapy. [2] Current pharmacological confirmed DCA well-known mechanism of action through inhibiting pyruvate dehydrogenase kinase (PDK). PDK inhibition causes stimulation of pyruvate dehydrogenase enzyme with subsequent increased conversion of glycolysis end products (pyruvate or lactate) into acetyl CoA with subsequent entry into Krebs cycle. [3] This frankly antagonizes cancer phenotype (Warburg effect) and deprives cancer cells of many lactate-based benefits e.g. cancer invasion, metastasis, angiogenesis, chemoresistance and radioresistance. [4] Ketone bodies are three compounds: acetone, acetoacetate and $\beta$-hydroxybutyrate. Ketone bodies are alternative energy substrates when glucose is not available (hypoglycemia of fasting or starvation) or when it is difficult to utilize glucose (diabetic ketoacidosis). Ketolysis is an energy-generating pathway that occurs outside the liver cells (extrahepatic). It occurs in peripheral tissues. [5] In this article, I prove my novel perspective that DCA antagonizes ketone bodies (acetoacetate and $\beta$-hydroxybutyrate) both structurally (Figure 1) and functionally to target cancer cells energetics through inhibiting ketolysis as a novel evidence-based mechanism of its anticancer effects.

\section{Ketolysis in Cancer Cells}

Ketone bodies oxidation (ketolysis) is an important source of energy to many cancer cells. Key enzyme of ketolysis has different names e.g. 3-oxoacid CoA-transferase-1 (OXCT1), acetoacetyl CoA transferase, 
succinyl-CoA 3-ketoacid CoA transferase (SCOT). SCOT (OXCT1) is the key enzyme in ketone body's metabolism that catalyzes the rate-determining step of ketolysis causing the formation of acetyl-CoA that finally starts the tricarboxylic acid cycle (Krebs cycle) for ATP production. OXCT1 participates in tumorigenesis and signaling in cancer cells where OXCT1 expression increases in different categories of cancer cells. [6] Normal hepatocytes (the site of ketone body synthesis) have no detectable OXCT1 protein due to the reported liver-specific silencing of the human gene encoding succinyl-CoA: OXCT1. [7] Ketone bodies, though produced in the liver, are not consumed in normal adult liver cells but are consumed as a source of energy in malignant liver cells.

Hepatocellular carcinoma was reported to redirect its energy pathways to ketolysis for progression under nutrition deprivation stress. That is a vital property in many cancer cells that distinguishes them from normal cells. OXCT1 expression is suppressed in normal adult liver tissues but is re-induced in hepatocellular carcinoma cells. Enhanced ketolysis in HCC protects hepatocellular carcinoma cells from excessive autophagy i.e. enhancing tumor growth. Analysis of clinical hepatocellular carcinoma samples reveals that increased OXCT1 expression predicts higher patient mortality. [8] Interestingly, twenty hepatocellular lesions displayed increased levels of OXCT1 (mRNA) and OXCT1 (protein). Interestingly, an aberrant ketolysis pathway was recently reported for continuous energy supply in cancer cells [9].

\section{DCA Antagonizes Ketone Bodies Oxidation}

Main energy generating reactions in human cells are oxidative phosphorylation (cell respiration), Krebs cycle, fatty acid oxidation (preceded by lipolysis), ketone bodies oxidation (ketolysis) and glycolysis (both aerobic and anaerobic). [5] The former four reactions are mitochondrial while the latter (glycolysis) is cytoplasmic. Glycolysis (both aerobic and anaerobic) is a vital energy source for aggressive, chemoresistant and radioresistant tumors where cancer cells mitochondria suffered from impaired functions that force cancer cells to be hyperglycolytic. [10] I report here a novel perspective that DCA targets both aerobic and anaerobic cancer cells. DCA targets anaerobic cancer cells through allowing lactate produced from tumors (Warburg effect) to be utilized in starting Krebs cycle (through PDK inhibition). DCA targets aerobic cancer cells by targeting some mitochondrial energy generating pathways e.g. ketolysis as I will prove here. DCA is a structural analog of acetoacetate and $\beta$-hydroxybutyrate (ketone bodies) (Figure 1). This gives the author the impression that DCA may exert its anticancer effects as a structural antagonist (antimetabolite) of ketone bodies (energy substrates) utilization and that DCA may act through targeting this energy generating pathway. Accumulating evidences support that DCA does not prevent formation of ketone bodies (ketogenesis) but DCA did prevent utilization of ketone bodies (ketolysis) [11] as a source of energy.

\section{DCA inhibits oxidation of acetoacetate and 3-hydroxybutyrate ( $\beta$ - hydroxybutyrate)}

Supporting the author's perspective that DCA antagonizes acetoacetate and ketone bodies was the early study by McAllister et al. who reported that DCA inhibits oxidation of acetoacetate, $\beta$-hydroxybutyrate and palmitate [11].

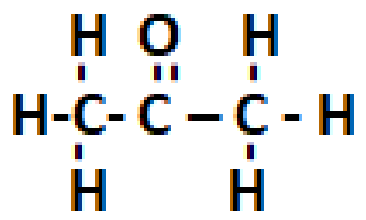

Acetone

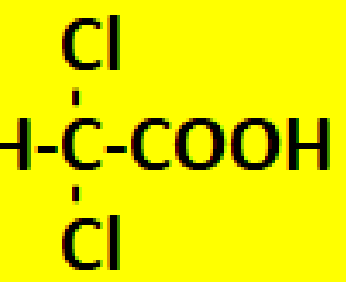

\section{Dichloroacetate}

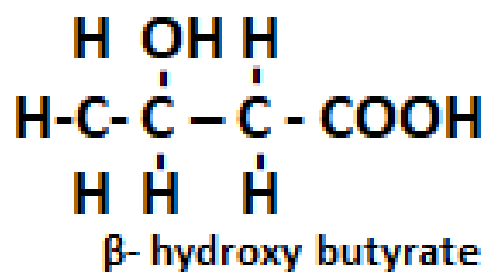

Figure 1. Dichloroacetate is a structural analog (and a possible competitor) of ketone bodies (acetoacetate and $\beta$-hydroxybutyrate) 


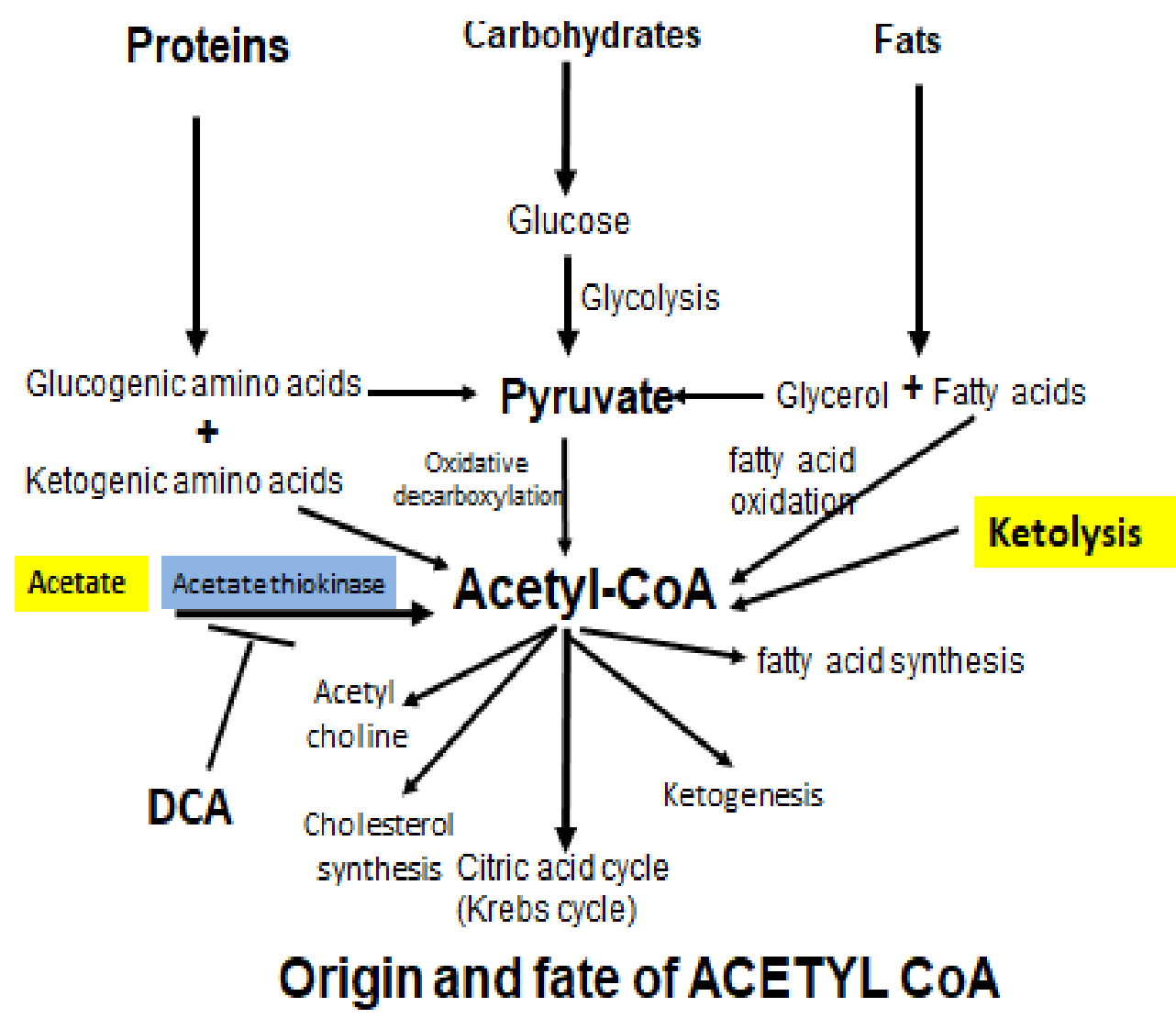

Figure 2. Sources and fate of active acetate (acetyl CoA). Acetyl CoA is produced during metabolism of proteins, lipids and carbohydrates. Acetyl CoA is also produced from acetic acid. Acetyl CoA is also produced during ketone bodies oxidation. Acetyl CoA starts Krebs cycle, helps in cholesterol synthesis and lipogenesis. All these are vital for cancer cells bioenergetics and synthesis of macromolecules

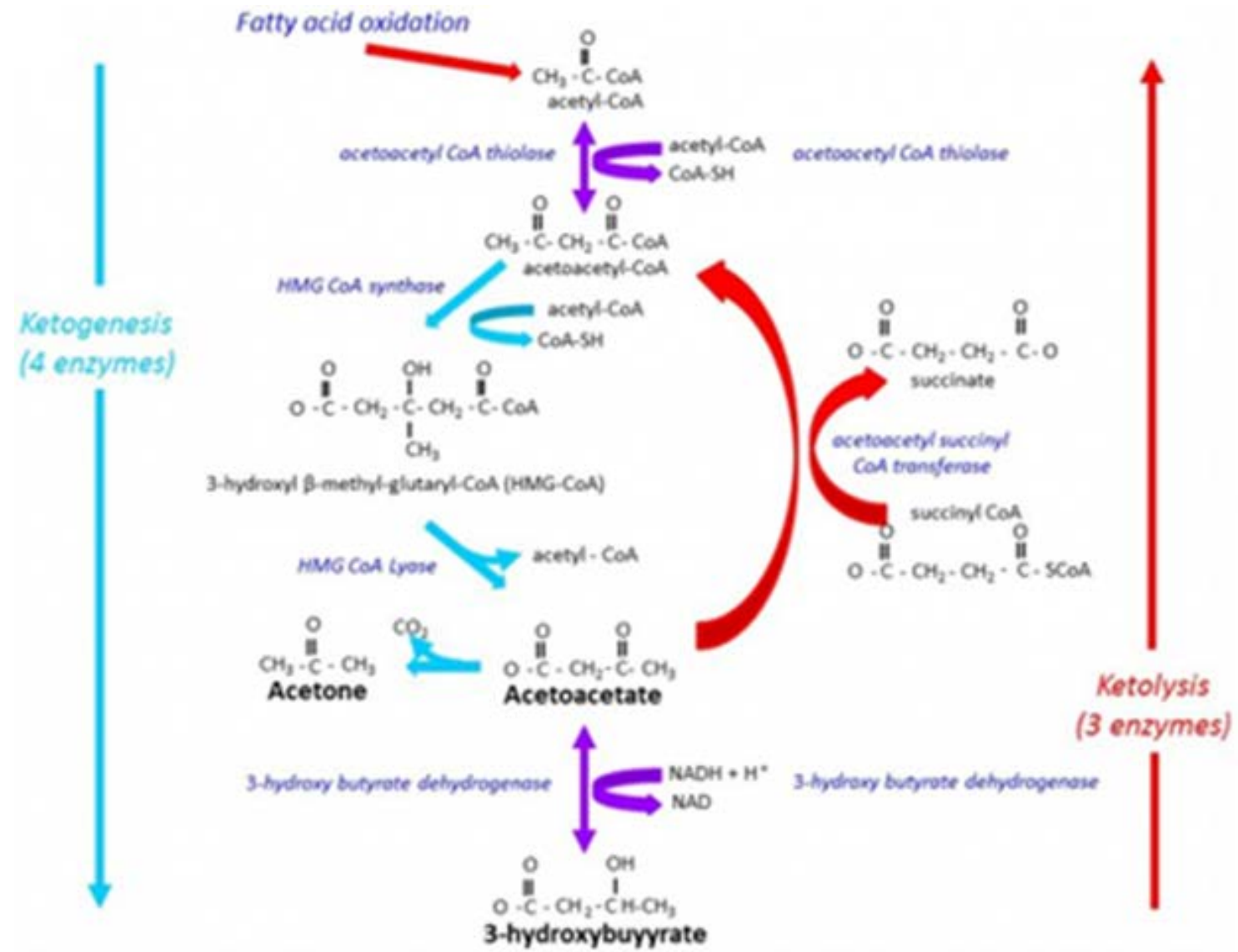

Figure 3. $\beta$-hydroxybutyrate (ketone body) is oxidized into acetoacetate (another ketone body) that is later converted into acetyl CoA. Dichloroacetate is a structural analog and a proved inhibitor of oxidation of both ketone bodies. This supports my view that dichloroacetate is an inhibitor of ketone bodies oxidation (ketolysis) 
Sources of the major energy substrate acetyl CoA include metabolism of the chief nutrients (carbohydrates, lipids and proteins). Oxidation of ketogenic amino acids and fatty acids gives acetyl CoA directly while glucogenic amino acids and glycerol utilize glycolysis pathway to form pyruvate as a source of acetyl CoA. Acetate itself is an energy substrate that can be converted into active acetate (acetyl CoA) that begins many energy generating pathways (Figure 2) [5].

\section{Evidences that DCA Inhibits Ketolysis}

DCA inhibits ketone bodies oxidation that gives a lot of energy (Figure 3). DCA was reported to inhibit $\beta$-hydroxybutyrate uptake by the extra-splanchnic tissues [12] and decrease clearance of ketone bodies. [12] Based on that, DCA may competitively antagonize ketone bodies. DCA infusion $(300 \mathrm{mg} / \mathrm{kg} / \mathrm{h})$ in $24 \mathrm{~h}$-starved rats for $4 \mathrm{~h}$ was reported to cause many biochemical changes: Significantly decreased blood glucose, significantly decreased plasma insulin, significantly decreased blood lactate and pyruvate concentrations but significantly increased concentrations of 3-hydroxybutyrate and acetoacetate. [12] This confirms the perspective that DCA inhibits ketone bodies utilization for energy production. For the author, this might be explained by structural antagonism between DCA and ketone bodies (acetoacetate and $\beta$-hydroxybutyrate). DCA was reported to inhibit $\beta$-hydroxybutyrate uptake by the extra-splanchnic tissues. That may be explained by structural antagonism leading to competitive uptake at target tissues. Importantly, DCA inhibited ketone bodies production in severe ketoacidosis and caused a marked decrease in blood ketone bodies concentrations in diabetic ketoacidosis rats [12,13].

Interestingly, DCA reduced blood glucose (caused hypoglycemia) through inhibiting hepatic glucose synthesis (gluconeogenesis) and stimulating glucose utilization by peripheral tissues. [14,15] This may force cancer cells to utilize alternative energy pathways e.g. ketolysis that is also inhibited by DCA. That collectively enhances DCA-induced energy failure in cancer cells and subsequent cancer cell death.

Moreover, DCA was reported to reduce ketonemia and ketonuria. DCA improves the lipid profile (inhibits hepatic triglyceride formation, inhibits cholesterol biosynthesis and decreases serum VLDL). DCA increased peripheral oxidation of alanine and lactate (depletes the Cori cycle that synthesizes glucose from lactate) causing depletion of the Warburg effect. [14,15]

\section{Conclusion}

Dichloroacetate is a promising anticancer drug that kills cancer cells through many different mechanisms among which is inhibition of ketone bodies oxidation.

\section{Conflict of Interest}

The author declares that there is no conflict of interest with anyone.

\section{Acknowledgements}

The author is grateful for Taibah university, Saudi Arabia for kindly providing the research facilities e.g. ithenticate programme.

\section{References}

[1] Dunbar EM, Coats BS, Shroads AL, Langaee T, Lew A, Forder JR Shuster JJ, Wagner DA, Stacpoole PW. Phase 1 trial of dichloroacetate (DCA) in adults with recurrent malignant brain tumors. Invest New Drugs. 2014 Jun; 32(3): 452-64.

[2] Strum SB, Adalsteinsson O, Black RR, Segal D, Peress NL, Waldenfels J. Case report: Sodium dichloroacetate (DCA) inhibition of the "Warburg Effect" in a human cancer patient: complete response in non-Hodgkin's lymphoma after disease progression with rituximab-CHOP. J Bioenerg Biomembr. 2013 Jun; 45(3):307-15.

[3] Tong J, Xie G, He J, Li J, Pan F, Liang H. Synergistic antitumor effect of dichloroacetate in combination with 5-fluorouracil in colorectal cancer. J Biomed Biotechnol. 2011; 2011: 740564

[4] El Sayed SM, Mohamed WG, Seddik MA, Ahmed AS, Mahmoud AG, Amer WH, Helmy Nabo MM, Hamed AR, Ahmed NS, AbdAllah AA. Safety and outcome of treatment of metastatic melanoma using 3-bromopyruvate: a concise literature review and case study. Chin J Cancer. 2014 Jul; 33(7): 356-64.

[5] Denise R. Ferrier Lippincott's illustrated reviews Biochemistry 6th edition. Wolters Klower. Lippincott Williams and Wilkins. 2014. Page 332

[6] Zhang S, Xie C. The role of OXCT1 in the pathogenesis of cancer as a rate-limiting enzyme of ketone body metabolism. Life Sci. 2017 Aug 15; 183: 110-115.

[7] Orii KE, Fukao T, Song XQ, Mitchell GA, Kondo N Liver-specific silencing of the human gene encoding succinylCoA: 3-ketoacid CoA transferase. Tohoku J Exp Med. 2008 Jul; 215(3): 227-36

[8] Huang, Li T, Wang L, Zhang L, Yan R, Li K, Xing S, Wu G, Hu L, Jia W, Lin SC, Dang CV, Song L, Gao P, Zhang $\mathrm{H}$. Hepatocellular carcinoma redirects to ketolysis for progression under nutrition deprivation stress. Cell Res. 2016 Oct; 26(10): 1112-1130.

[9] Galluzzi L, Kroemer G. Aberrant ketolysis fuels hepatocellular cancer progression. Cell Res. 2016 Oct; 26(10):1077-1078.

[10] Ferreira LM. Cancer metabolism: the Warburg effect today. Exp Mol Pathol. 2010 Dec; 89(3): 372-80.

[11] McAllister A, Allison SP, Randle PJ. Effects of dichloroacetate on the metabolism of glucose, pyruvate, acetate, 3-hydroxybutyrate and palmitate in rat diaphragm and heart muscle in vitro and on extraction of glucose, lactate, pyruvate and free fatty acids by dog heart in vivo. Biochem J. 1973 Aug; 134(4): 1067-81.

[12] Blackshear PJ, Holloway PA, Alberti KG. The metabolic effects of sodium dichloroacetate in the starved rat. Biochem J. 1974 Aug; 142(2): 279-86.

[13] Blackshear PJ, Holloway PA, Alberti KG. Metabolic interactions of dichloroacetate and insulin in experimental diabetic ketoacidosis. Biochem J. 1975 Feb; 146(2): 447-56.

[14] Stacpoole PW, Greene YJ. Dichloroacetate. Diabetes Care. 1992 Jun; 15(6): 785-91.

[15] Stacpoole PW, Henderson GN, Yan Z, James MO. Clinical pharmacology and toxicology of dichloroacetate. Environ Health Perspect. 1998 Aug; 106 Suppl 4: 989-94. 